OPEN ACCESS

Edited by:

Margaret Ip

The Chinese University of Hong Kong, China

Reviewed by:

Zisis Kozlakidis,

International Agency for Research on

Cancer (IARC), France

Pedro Xavier-Elsas,

Federal University of

Rio de Janeiro, Brazil

*Correspondence:

Chris Kenyon

ckenyon@itg.be

Specialty section:

This article was submitted to Infectious Diseases-Surveillance,

Prevention and Treatment,

a section of the journal

Frontiers in Public Health

Received: 22 February 2019

Accepted: 28 May 2019

Published: 12 June 2019

Citation:

Kenyon C (2019) Toward a Set of Criteria to Decide Which STIs to

Screen for in PrEP Cohorts.

Front. Public Health 7:154

doi: 10.3389/fpubh.2019.00154

\section{Toward a Set of Criteria to Decide Which STIs to Screen for in PrEP Cohorts}

\author{
Chris Kenyon ${ }^{1,2 *}$ \\ ${ }^{1}$ HIVISTI Unit, Institute of Tropical Medicine, Antwerp, Belgium, ${ }^{2}$ Division of Infectious Diseases and HIV Medicine, University \\ of Cape Town, Cape Town, South Africa
}

Contemporary HIV preexposure prophylaxis (PrEP) cohorts are characterized by high rates of partner change and as a result have high and fairly stable prevalences of N. gonorrhoeae and C. trachomatis. The available evidence suggests that intensive 3-monthly screening in this setting does not have a large effect on the prevalence of these infections but results in high antimicrobial exposures. Gonorrhea/chlamydia screening may thus be doing more harm than good. Compelling arguments can, however, be made to screen for HIV, hepatitis C, and syphilis in PrEP cohorts. In this perspective piece, we explore the logical basis for deciding which STIs to screen for in PrEP cohorts. We propose that a Delphi consensus methodology is used to derive, assess, and apply a broadly accepted set of criteria to evaluate which STIs to screen for in these cohorts. Finally, to illustrate the utility of the process, we derive and apply our own list of criteria as to which STIs to screen for. This process leads to a controversial conclusion, namely that stopping gonorrhea/chlamydia screening in a controlled and phased manner may offer net health benefits to PrEP cohorts.

Keywords: STI screening, antimicrobial resistance, gonorrhea, chlamydia, M. genitalium, MSM, PrEP, Delphi consensus

\section{INTRODUCTION}

Preexposure prophylaxis (PrEP) refers to the use of antiretroviral medications to prevent HIVinfection in persons at high risk of HIV acquisition (1). One of the largest target PrEP populations are men who have sex with men (MSM) with high rates of partner change (1-3). The participants in the PrEP study typically report a mean of between 9.3 and 18 partners per 3 months $(1,4,5)$. The combination of high rates of partner change and infrequent condom usage (particularly for oral sex) result in high equilibrium prevalence of a range of STIs including Neisseria gonorrhoeae, Chlamydia trachomatis, and Mycoplasma genitalium. As a result, PrEP guidelines commonly recommend frequent (3-monthly) and 3-site (anorectal, pharyngeal, and urethral) screening for gonorrhea/chlamydia-termed $3 \times 3$ screening (6-9). A recent systematic review found that intense screening was not associated with a decline in the prevalence of these infections-regardless of whether the screening was conducted every 3,6 , or 12 months (7). The high prevalence of N. gonorrhoeae and C. trachomatis in PrEP cohorts, however, means that screening for these infections (and treating all positives which is standard practice) exposes around 20\% of PrEP recipients to antimicrobials every 3 months (10). This results in macrolide exposures of up to 4,400 
standard doses/1,000 population per year (10). This exposure level is 41 times greater than that of populations such Latvia and considerably higher than exposure levels found to be strongly associated with the induction of antimicrobial resistance (AMR) in a range of bacteria including $N$. gonorrhea and Treponema pallidum (10-15).

A range of different studies have revealed that a key way to prevent the emergence of AMR is reducing the consumption of antimicrobials to below resistance inducing thresholds (14, 16). Populations that have kept antimicrobial consumption below such thresholds tend to have low rates of AMR for a range of bug-drug combinations whereas populations with high antimicrobial exposures tend to have high rates of AMR (10$15)$. In the case of syphilis, for example, macrolide resistance in Treponema pallidum increased to between 65 and $100 \%$ in populations exposed to more than 700 doses of macrolides $/ 1,000$ population per year but was almost universally non-existent in populations whose macrolide consumption was less than this threshold (11). Likewise populations with high consumption of cephalosporins, macrolides and fluroroquinolones have a higher prevalence of homologous AMR in circulating $N$. gonorrhoeae than lower consumption populations $(15,17)$. In a similar vein, in-vitro studies since the 1940's have demonstrated that for a range of microbes (including $N$. gonorrhoeae) resistance to a particular antimicrobial rapidly follows sustained exposure to that antimicrobial (18). An important conclusion of the studies linking AMR to antimicrobial consumption has been to make antimicrobial stewardship (limiting the usage of antimicrobials to indications where there is good evidence of net-benefit) a central pillar of the strategy to retard the emergence of AMR (16).

Because approximately $90 \%$ of gonorrhea and chlamydia infections are asymptomatic and self-limiting in MSM, roughly $90 \%$ of infections would not result in antimicrobial exposure in the absence of screening $(19,20)$. Thus, screening for these infections in MSM results in a substantial exposure to antimicrobials. This consideration, in conjunction with the emerging threat of untreatable STIs motivates us to interrogate what the evidence base is for recommending gonorrhea/chlamydia screening in this setting (16)? As far as we are aware, there are no randomized controlled trials that have assessed the efficacy of screening for any STI in MSM PrEP cohorts (7). Despite this, very cogent arguments could be made for screening for hepatitis C, HIV and syphilis but not Mycoplasma genitalium (21) in these cohorts. In this perspective piece, we explore the logical basis for making this distinction. We start with a brief review of studies that investigate the evidence for and against gonorrhea/chlamydia screening in this setting. We then propose that a Delphi consensus methodology is used to derive, assess and apply a broadly accepted set of criteria to evaluate which STIs to screen for in MSM PrEP cohorts. Finally, to illustrate the utility of the process, we derive and apply our own list of criteria as to which STIs to screen for. This process leads to the conclusion that stopping gonorrhea/chlamydia screening may offer net health benefits to MSM in PrEP cohorts.

\section{EVIDENCE FOR AND AGAINST SCREENING GONORRHEA/CHLAMYDIA}

Intensive gonorrhea/chlamydia screening in PrEP cohorts has been hypothesized to provide a number of benefits including reducing the prevalence of these infections, reducing HIV transmission and engaging higher risk individuals in care $(7,22)$. On the other hand, intensive screening could induce AMR in these and other bacteria, it is costly and it may result in a certificate of health effect whereby those screened may feel that if they come for regular screening this gives them a bill of health that means they can relax safety devices perceived to be onerous such as condoms $(2,7,10,23)$.

None of these benefits and harms have been established empirically. In particular no randomized controlled trials have been conducted in MSM to evaluate the risks and benefits of gonorrhea/chlamydia screening (7). In heterosexuals the evidence of the efficacy of screening is mixed $(24,25)$. Even if the evidence in heterosexuals was strong this could not be assumed to apply to MSM PrEP cohorts due to a number of factors including a higher sexual network connectivity in this population (2). In its systematic review to inform chlamydia/gonorrhea screening guidelines, the United States Preventive Services Task Force (USPSTF) found no relevant randomized controlled trials in men and concluded: "the current evidence is insufficient to assess the balance of benefits and harms of screening for chlamydia and gonorrhea in men" (7).

Since the publication of the USPSTF's systematic review and guideline a number of relevant studies have been published. A systematic review of observational studies assessing the association between the intensity of gonorrhea/chlamydia screening and prevalence in MSM found no association between screening overall or the intensity of screening and a reduction in the prevalence of gonorrhea or chlamydia (7).

Recently published modeling studies have reached slightly different conclusions as to the efficacy of screening in MSM. One modeling study, that ignored transmission to and from the pharynx of gonorrhea and chlamydia, found that intense screening in MSM PrEP recipients in the United States, could halve the prevalence of these infections (26). A different modeling study of Belgian MSM, that included pharyngeal transmission, suggested much more moderate effects of screening (27). A key finding of both these studies was that it was the high sexual network connectivity which generated the high prevalence of gonorrhea and chlamydia.

There are wide variations in the intensity of self-reported gonorrhea/chlamydia screening in MSM between countries within Europe. An ecological study found no association between country-level screening intensity and the prevalence or incidence of these two infections (28).

Finally, a number of studies have suggested that intensive screening may be a risk factor for AMR. One analysis found that $3 \times 3$ screening in PrEP cohorts results in macrolide exposures that are strongly associated with the induction of AMR in a range of bacteria (10). Two ecological analyses have found positive associations between the intensity of 
TABLE 1 | Non-exclusive list of possible criteria for evaluating net utility of screening six specific STIs in MSM PrEP cohorts".

\begin{tabular}{|c|c|c|c|c|c|c|}
\hline & $\mathrm{Ng}$ & Mg & Ct & Tp & HCV & HIV \\
\hline \multicolumn{7}{|c|}{ STEP 1: ASSESS IF HOST-PATHOGEN INTERACTIONS ARE AMENABLE TO SCREENING } \\
\hline 1. Undetected infection typically associated with serious adverse clinical outcomes & 1 & 1 & 1 & 4 & 4 & 5 \\
\hline 2. Long period between infection and disease onset & 1 & 1 & 1 & 3 & 5 & 5 \\
\hline 3. Not spontaneously cleared by immune system & 0 & 0 & 0 & 3 & 3 & 5 \\
\hline 4. No immunity from naturally cleared infection & 3 & 3 & 2 & 2 & 5 & 5 \\
\hline Total & 5 & 5 & 2 & 12 & 17 & 20 \\
\hline \multicolumn{7}{|l|}{ STEP 2: ASSESS THE RISK OF INDUCING AMR } \\
\hline 1. Low risk of inducing AMR in pathogen itself given standard therapy & 0 & 0 & 4 & 4 & 5 & 5 \\
\hline 2. Low risk of inducing AMR in microbiome given standard therapy & 0 & 1 & 2 & 4 & 5 & 5 \\
\hline Total & 0 & 1 & 6 & 8 & 10 & 10 \\
\hline
\end{tabular}

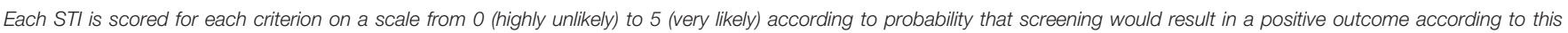
criterion"

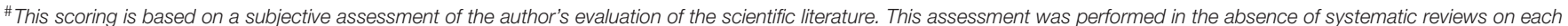
of these criteria/pathogen combinations.

Ng, Neisseria gonorrhoeae; Mg, Mycoplasma genitalium; Ct, Chlamydia trachomatis; Tp, Treponema pallidum; HCV, hepatitis C; HIV, Human Immunodeficiency Virus.

gonorrhea/chlamydia screening and the prevalence of AMR in N. gonorrhoeae $(29,30)$.

\section{WHAT CRITERIA SHOULD BE USED TO DECIDE WHICH STIS TO SCREEN FOR IN MSM PREP COHORTS?}

Which criteria should be used to evaluate if screening for a particular STI in PrEP cohorts is advisable or not? A first step is evaluating if screening for the STI in question meets the modified World Health Organization criteria for screening (31). In particular, one would need to establish that the overall benefits of screening outweigh the harms (31). Whilst randomized controlled trials would be the optimal way to answer this question, to the best of our knowledge, none are planned. We do, however, have a reasonable amount of information which could be used to better guide which STIs to screen for. This includes the epidemiological evidence reviewed above as well as information pertaining to the basic biology of each STI, host immune responses and the resistogenicity of STI treatments. Ideally, a group of experts and stakeholders could be brought together and, using a Delphi-consensus-type process, develop a set of criteria to evaluate which STIs to screen for in MSM PrEP populations (32). A suitable and widely used methodology that could be used to conduct this process would be the Grading of Recommendations Assessment, Development and Evaluation (GRADE) evidenceto-decision process $(33,34)$. In such a process the experts would first select a set of criteria to use to evaluate which STIs to screen for in MSM PrEP cohorts. In the second phase, they would review the relevant literature to decide to what extent the evidence supports screening per criterion and STI (Table 1). In each phase the experts would provide their initial answers which are then summarized, anonymized and shared with the group by a facilitator. The experts are then invited to revise their answers and the process repeated until either consensus is reached or a pre-specified outcome is attained $(32,35)$. A similar process was successfully used to derive the widely used new clinical criteria for defining septic shock (32).

\section{AN ILLUSTRATION OF POSSIBLE STI-SCREENING-CRITERIA}

Whilst this Delphi-process is considerably beyond the scope of this opinion piece, we illustrate how the criteria selection phase may unfold by outlining the criteria which we would select. We would choose a two-step, 6-criteria process for evaluating which STIs to screen for (Table 1). Based on our reading of the literature, we then provide a score for each of these 6 criteria relating to each of 6 STIs-chlamydia, HIV, hepatitis C, gonorrhea, M. genitalium, and syphilis. Each is scored from 0 to 5 according to whether they are highly unlikely (scored 0 ) to very likely (scored 5) to result in a net utility for screening (Table $\mathbf{1}$ ).

\section{Step 1: Assess if the Biology of STI-host Interactions Make the STI Amenable to Screening}

Numerous aspects of the way $N$. gonorrhoeae circulates in MSM decreases the probability that screening will be beneficial. Symptomatic disease is thought to typically occur soon $(2-21$ days) after infection. If symptoms do not develop, the infection (particularly in the pharynx and rectum) tends to persist in a low abundance state for up to 6 months (20). Highly exposed individuals develop a type-specific immunity but this immunity is largely ineffective in low exposure individuals $(20,36)$. The vast majority of $N$. gonorrhoeae infections are asymptomatic and selflimiting in this population $(19,20)$. Screening is far more likely to diagnose infections in the 6 month asymptomatic tail phase (when $N$. gonorrhoeae abundance is likely lower and therefore less infectious) than in the acute first weeks post infection. These features reduce the probability that screening will decrease either symptomatic infections or N. gonorrhoeae transmissionsassuming that the low abundance infections are less infectious. 


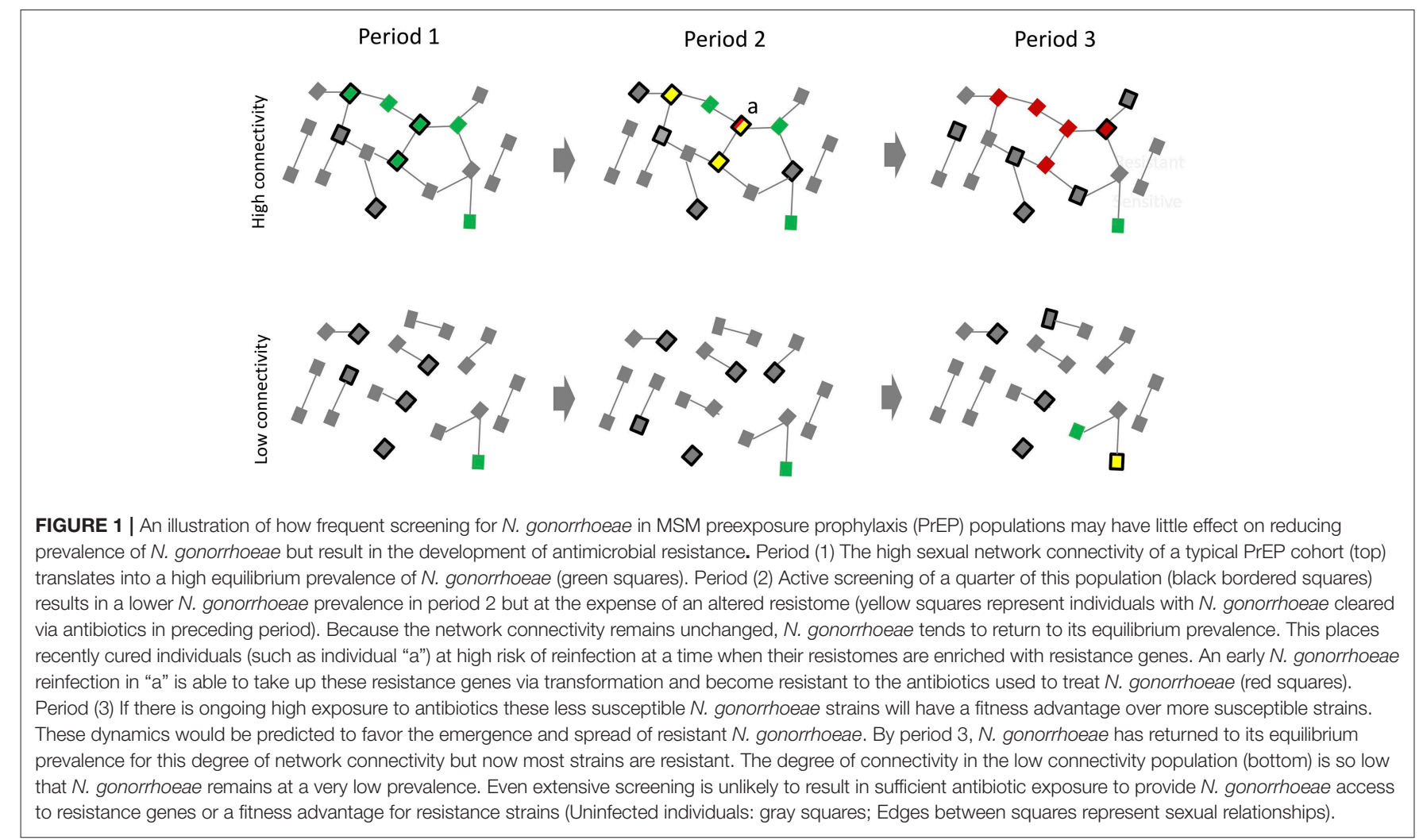

Similar considerations apply to C. trachomatis and M. genitalium. In the case of $C$. trachomatis there is however better evidence that treatment of C. trachomatis results in "arrested immunity" and thereby paradoxically increases the probability of reinfection and may even lead to increases in prevalence $(37,38)$. As a result, all 3 of these STIs score poorly for being amenable to screening when assessed by these amenability criteria (Table 1).

The assessments for syphilis, hepatitis C and HIV are, however, very different. Each has a relatively long latent period, each results in serious disease and the probability of spontaneous clearance ranges from moderate (syphilis) to close to zero (HIV) (39-41). As a result, screening is likely to both reduce the probability of serious disease in infected individuals and the probability of onward transmission. They thus have high screening-utility-scores for the first step (Table 1).

\section{Step 2: Assess the Risk of Inducing AMR}

As noted above, gonorrhea/chlamydia screening in MSM can result in exposure levels to macrolides/cephalosporins that are strongly associated with resistance in N. gonorrhoeae, Treponema pallidum and a range of other bacteria. Macrolides are typically recommended as first line therapy for M. genitalium, but involve a $10 \%$ risk of inducing macrolide AMR in M. genitalium per treatment (42). These considerations suggest that screening for $N$. gonorrhoeae and M. genitalium run a high risk of inducing AMR in both the bacteria themselves and the resident microbiomes. A single dose of azithromycin, for example, has been shown to have adverse effects on the recipient's microbiome and resistome (including macrolide resistance mechanisms) that persists for up to 6 months in the oropharynx and 48 months in the colon $(43,44)$. If an azithromycin recipient acquires a new $N$. gonorrhoeae infection in this time period, it could acquire the macrolide resistance mutations from the commensal population (via horizontal gene transfer) and thereby become less-susceptible to macrolides (Figure 1) (2, 45). Various studies have provided suggestive evidence that $N$. gonorrhoeae has acquired penicillin, cephalosporin, and macrolide resistance via this type of mechanism $(45,46)$. As a result, $N$. gonorrhoeae and M. genitalium once again score poorly in the second step criteria (Table 1).

Screening for hepatitis C and HIV, on the other hand, involves little risk of these pathogens and commensals acquiring resistance if standard treatment protocols are followed. Likewise, if penicillin is used for therapy, screening for syphilis involves an extremely low risk of inducing AMR in T. pallidum and a lower risk of inducing wide-ranging resistance in the microbiome than other antimicrobial classes such as the macrolides and extended spectrum cephalosporins $(39,47)$. As a result, these three STIs have high scores for screening in both steps one and two.

If these two steps are followed, then a strong case can be made that screening for T. pallidum, hepatitis C and HIV is likely to reduce disease in individuals screened, reduce transmission and involve little or no risk of inducing AMR. Conversely, screening for N. gonorrhoeae, C. trachomatis and M. genitalium is less likely to result in decreasing disease in those screened, less likely to reduce prevalence and particularly for $N$. gonorrhoeae and $M$. 
genitalium, more likely to induce AMR. Modeling studies that include the effect of screening on (1) prevalence/incidence of each STI, (2) the probability of AMR emergence and (3) costeffectiveness analyses would be useful to provide further evidence as to the net utility of screening. The use of antimicrobials has also been associated with the genesis of AMR in pathobionts and commensals not targeted by the antimicrobials (bystander selection) (48). Antimicrobials also have a range of other deleterious effects such as on the health of the microbiome which may be long lasting. Optimally studies evaluating the net utility of screening should include these as secondary outcomes.

\section{CONCLUSION}

PrEP populations typically have high rates of partner change, partner concurrency and a low prevalence of condom use $(1,5)$. These behaviors generate dense sexual networks which result in high equilibrium prevalences of most STIs [reviewed in (2), (49); Figure 1]. The prevalence of $N$. gonorrhoeae, $C$. trachomatis and $M$. genitalium, for example, are each typically $8-17 \%$ in PrEP cohorts $(7,50)$. The sexual networks in these cohorts are so dense that even $3 \times 3$ screening/treatment for $N$. gonorrhoeae, C. trachomatis, and $M$. genitalium has not been found to result in a decrease in the prevalence of these infections $(5,7,9,27,50)$. Screening does however result in considerable increases in antimicrobial exposure with the

\section{REFERENCES}

1. Grant RM, Lama JR, Anderson PL, McMahan V, Liu AY, Vargas L, et al. Preexposure chemoprophylaxis for HIV prevention in men who have sex with men. N Engl J Med. (2010) 363:2587-99. doi: 10.1056/NEJMoa 1011205

2. Kenyon CR, Schwartz IS. Effects of sexual network connectivity and antimicrobial drug use on antimicrobial resistance in Neisseria gonorrhoeae. Emerg Infect Dis. (2018) 24:1195-203. doi: 10.3201/eid2407.172104

3. Kenyon C, Osbak K. Certain attributes of the sexual ecosystem of high-risk MSM have resulted in an altered microbiome with an enhanced propensity to generate and transmit antibiotic resistance. Med Hypotheses. (2014) 83:196202. doi: 10.1016/j.mehy.2014.04.030

4. Liu AY, Cohen SE, Vittinghoff E, Anderson PL, Doblecki-Lewis S, Bacon O, et al. Preexposure prophylaxis for HIV infection integrated with municipaland community-based sexual health services. JAMA Intern Med. (2016) 176:75-84. doi: 10.1001/jamainternmed.2015.4683

5. Traeger MW, Schroeder SE, Wright EJ, Hellard ME, Cornelisse VJ, Doyle JS, et al. Effects of pre-exposure prophylaxis for the prevention of human immunodeficiency virus infection on sexual risk behavior in men who have sex with men: a systematic review and meta-analysis. Clin Infect Dis. (2018) 67:676-86. doi: 10.1093/cid/ciy182

6. Tan DHS, Hull MW, Yoong D, Tremblay C, O’Byrne P, Thomas $\mathrm{R}$, et al. Canadian guideline on HIV pre-exposure prophylaxis and nonoccupational postexposure prophylaxis. CMAJ. (2017) 189:E1448-58. doi: $10.1503 / \mathrm{cmaj} .170494$

7. Tsoumanis A, Hens N, Kenyon CR. Is screening for chlamydia and gonorrhea in men who have sex with men associated with reduction of the prevalence of these infections? A systematic review of observational studies. Sex Transm Dis. (2018) 45:615-22. doi: 10.1097/OLQ.0000000000000824

8. Centers for Disease Control and Prevention: US Public Health Service. Preexposure Prophylaxis for the Prevention of HIV Infection in the United States-2017 Update: A Clinical Practice Guideline. Available online attending risk of AMR (10). These considerations suggest the need to revisit the evidence base for screening for these infections. The urgency for this stems from predictions such as those from the O'Niell report that antimicrobial resistant infections will cause more deaths worldwide than cancer in 2050 (16). This is not inevitable. We know that the predominant determinant of the AMR epidemic is excess use of antimicrobials (14, 16). Populations with low consumption of antimicrobials have corresponding low prevalences of AMR (12-15). If providers and MSM PrEP clients consider the risks and benefits of screening for gonorrhea/chlamydia via a Delphi-consensus process they may jointly decide to continue screening for HIV, hepatitis $\mathrm{C}$ and syphilis but reduce or stop screening for gonorrhea/chlamydia. One option would be to phase out gonorrhea/chlamydia screening in a controlled and staggered way as part of a pragmatic study design. This would provide further evidence on the net benefits and risks of screening.

\section{DATA AVAILABILITY}

No datasets were generated or analyzed for this study.

\section{AUTHOR CONTRIBUTIONS}

The author confirms being the sole contributor of this work and has approved it for publication. at: https://wwwcdcgov/hiv/pdf/risk/prep/cdc-hiv-prep-guidelines-2017pdf. 2018 (accessed December 20, 2018).

9. De Baetselier I, Reyniers T, Nöstlinger C, Wouters K, Fransen K, Crucitti T, et al. Pre-Exposure Prophylaxis (PrEP) as an additional tool for HIV prevention among men who have sex with men in Belgium: the Be-PrEP-ared study protocol. JMIR Res Protoc. (2017) 6:e11. doi: 10.2196/resprot.6767

10. Kenyon $\mathrm{C}$. We need to consider collateral damage to resistomes when we decide how frequently to screen for chlamydia/gonorrhoea in PrEP cohorts. AIDS. (2019) 33:155-7. doi: 10.1097/QAD.0000000000002020

11. Kenyon C. Prevalence of macrolide resistance in Treponema pallidum is associated with macrolide consumption. J Med Microbiol. (2018) 68:119-23. doi: 10.1099/jmm.0.000885

12. Levy SB, Marshall B. Antibacterial resistance worldwide: causes, challenges and responses. Nat Med. (2004) 10(Suppl. 12):S122-9. doi: 10.1038/nm1145

13. Bell BG, Schellevis F, Stobberingh E, Goossens H, Pringle M. A systematic review and meta-analysis of the effects of antibiotic consumption on antibiotic resistance. BMC Infect Dis. (2014) 14:13. doi: 10.1186/1471-2334-14-13

14. Goossens H, Ferech M, Vander Stichele R, Elseviers M; ESAC Project Group. Outpatient antibiotic use in Europe and association with resistance: a cross-national database study. Lancet. (2005) 365:579-87. doi: 10.1016/S0140-6736(05)17907-0

15. Kenyon C, Buyze J, Wi T. Antimicrobial consumption and susceptibility of Neisseria gonorrhoeae: a global ecological analysis. Front Med. (2018) 5:329. doi: 10.3389/fmed.2018.00329

16. O’Neill J. Tackling Drug-Resistant Infections Globally: Final Report and Recommendations. HM Government and Welcome Trust (2018).

17. Kenyon C, Buyze J, Spiteri G, Cole MJ, Unemo M. Antimicrobial consumption is associated with decreased antimicrobial susceptibility of Neisseria gonorrhoeae in 24 European countries: an ecological analysis. J Infect Dis. (2019). doi: 10.1093/infdis/jiz153. [Epub ahead of print].

18. Morin A, Saheb SA, Bisaillon JG, Beaudet R, Sylvestre M. Development in vitro of penicillin-resistant strains of the gonococcus. Exp Biol Med. (1945) 1:62-9. doi: 10.3181/00379727-58-14825 
19. Fairley CK, Hocking JS, Zhang L, Chow EP. Frequent transmission of gonorrhea in men who have sex with men. Emerg Infect Dis. (2017) 23:102-4. doi: 10.3201/eid2301.161205

20. Hook EW, Handsfield H. Gonococcal infections in the adult In: Holmes KK, editor. Sexually Transmitted Diseases. 3rd ed. New York, NY: McGraw-Hill, Health Professions Division (1999). p. 1118.

21. Horner P, Donders G, Cusini M, Gomberg M, Jensen JS, Unemo M. Should we be testing for urogenital Mycoplasma hominis, Ureaplasma parvum and Ureaplasma urealyticum in men and women? - a position statement from the European STI Guidelines Editorial Board. J Eur Acad Dermatol. (2018) 32:1845-51. doi: 10.1111/jdv.15146

22. Ridpath AD, Chesson H, Marcus JL, Kirkcaldy RD, Torrone EA, Aral SO, et al. Screening Peter to Save Paul: the population-level effects of screening men who have sex with men for gonorrhea and chlamydia. Sex Transm Dis. (2018) 45:623-25. doi: 10.1097/OLQ.0000000000000892

23. Stewart-Brown S, Farmer A. Screening could seriously damage your health. BMJ. (1997) 314:533-4. doi: 10.1136/bmj.314.7080.533

24. Hocking JS, Temple-Smith M, Guy R, Donovan B, Braat S, Law M, et al. Population effectiveness of opportunistic chlamydia testing in primary care in Australia: a cluster-randomised controlled trial. Lancet. (2018) 392:1413-22. doi: 10.1016/S0140-6736(18)31816-6

25. Low N, Redmond S, Uuskula A, van Bergen J, Ward H, Andersen B, et al. Screening for genital chlamydia infection. Cochrane Database Syst Rev. (2016) 9:CD010866. doi: 10.1002/14651858.CD010866.pub2

26. Jenness SM, Weiss KM, Goodreau SM, Gift T, Chesson H, Hoover KW, et al. Incidence of gonorrhea and chlamydia following HIV preexposure prophylaxis among men who have sex with men: a modeling study. Clin Infect Dis. (2017) 65:712-18. doi: 10.1093/cid/cix439

27. Buyze J, Vandenberghe W, Hens N, Kenyon C. Current levels of gonorrhoea screening in MSM in Belgium may have little effect on prevalence: a modelling study. Epidemiol Infect. (2018) 146:333-8. doi: 10.1017/S09502688180 00092.

28. Kenyon C. Screening is not associated with reduced incidence of gonorrhoea or chlamydia in MSM; an ecological study of 23 European countries. F1000Research. (2019) 8:160. doi: 10.12688/f1000research.17955.1

29. Kenyon CR, Kenyon CR. Association between intensity of STI screening and development of antimicrobial resistance in $N$. gonorrhoeae in 12 cities in the USA: an ecological study. F1000Research. (2018) 7:1237-44. doi: 10.12688/f1000research.15569.1

30. Kenyon CR, De Baetselier I, Crucitti T. Does gonorrhoea screening intensity play a role in the early selection of antimicrobial resistance in men who have sex with men (MSM)? A comparative study of Belgium and the United Kingdom. F1000Research. (2018) 77:569. doi: 10.12688/f1000research.14869.1

31. Andermann A, Blancquaert I, Beauchamp S, Déry V. Revisiting Wilson and Jungner in the genomic age: a review of screening criteria over the past 40 years. B World Health Organ. (2008) 86:317-19. doi: 10.2471/BLT.07.050112

32. Shankar-Hari M, Phillips GS, Levy ML, Seymour CW, Liu VX, Deutschman CS, et al. Developing a new definition and assessing new clinical criteria for septic shock: For the Third International Consensus Definitions for Sepsis and Septic Shock (Sepsis-3). JAMA. (2016) 315:775-87. doi: $10.1001 /$ jama.2016.0289

33. Schünemann HJ, Oxman AD, Akl EA, Brozek JL, Montori VM, Heffner J, et al. Moving from evidence to developing recommendations in guidelines: article 11 in Integrating and coordinating efforts in COPD guideline development. An official ATS/ERS workshop report. Proc Am Thorac Soc. (2012) 9:282-92. doi: 10.1513/pats.201208-064ST

34. Schünemann HJ, Wiercioch W, Brozek J, Etxeandia-Ikobaltzeta I, Mustafa RA, Manja V, et al. GRADE Evidence to Decision (EtD) frameworks for adoption, adaptation, and de novo development of trustworthy recommendations: GRADE-ADOLOPMENT. J Clin Epidemiol. (2017) 81:101-10. doi: 10.1016/j.jclinepi.2016.09.009

35. Rowe G, Wright G. The Delphi technique as a forecasting tool: issues and analysis. Int $J$ Forecasting. (1999) 15:353-75. doi: 10.1016/S0169-2070(99)00018-7
36. Plummer FA, Simonsen JN, Chubb H, Slaney L, Kimata J, Bosire M, et al. Epidemiologic evidence for the development of serovar-specific immunity after gonococcal-infection. J Clin Investig. (1989) 83:1472-6. doi: 10.1172/JCI114040

37. Geisler WM, Lensing SY, Press CG, Hook EW III. Spontaneous resolution of genital Chlamydia trachomatis infection in women and protection from reinfection. J Infect Dis. (2013) 207:1850-6. doi: 10.1093/infdis/jit094

38. Omori R, Chemaitelly H, Althaus CL, Abu-Raddad LJ. Does infection with Chlamydia trachomatis induce long-lasting partial immunity? Insights from mathematical modelling. Sex Transm Infect. (2018) 95:sextrans-2018-053543. doi: 10.1136/sextrans-2018-053543

39. Tramont E. Syphilis. In: Mandell GL, Bennett JE, Dolin R, editors. Principles and Practice of Infectious Diseases, 8th ed. Philadelphia, PA: Churchill Livingstone Inc (2015) 2684-709.

40. Thomas DL, Seeff LB. Natural history of hepatitis C. Clin Liver Dis. (2005) 9:383-398, vi. doi: 10.1016/j.cld.2005.05.003

41. Moore RD, Chaisson RE. Natural history of HIV infection in the era of combination antiretroviral therapy. AIDS. (1999) 13:1933-42. doi: 10.1097/00002030-199910010-00017

42. Golden MR, Workowski KA, Bolan G. Developing a public health response to Mycoplasma genitalium. J Infect Dis. (2017) 216(Suppl. 2):S420-6. doi: 10.1093/infdis/jix200

43. Malhotra-Kumar S, Lammens C, Coenen S, Van Herck K, Goossens H. Effect of azithromycin and clarithromycin therapy on pharyngeal carriage of macrolide-resistant streptococci in healthy volunteers: a randomised, double-blind, placebo-controlled study. Lancet. (2007) 369:48290. doi: 10.1016/S0140-6736(07)60235-9

44. Jakobsson HE, Jernberg C, Andersson AF, Sjölund-Karlsson M, Jansson JK, Engstrand L. Short-term antibiotic treatment has differing long-term impacts on the human throat and gut microbiome. PLoS ONE. (2010) 5:e9836. doi: 10.1371/journal.pone.0009836

45. Wadsworth CB, Arnold BJ, Sater MRA, Grad YH. Azithromycin resistance through interspecific acquisition of an epistasis-dependent efflux pump component and transcriptional regulator in Neisseria gonorrhoeae. MBio. (2018) 9:e01419-18. doi: 10.1128/mBio.01419-18

46. Ito M, Deguchi T, Mizutani KS, Yasuda M, Yokoi S, Ito S, et al. Emergence and spread of Neisseria gonorrhoeae clinical isolates harboring mosaic-like structure of penicillin-binding protein 2 in central Japan. Antimicrob Agents Chemother. (2005) 49:137-43. doi: 10.1128/AAC.49.1.137-143.2005

47. Langdon A, Crook N, Dantas G. The effects of antibiotics on the microbiome throughout development and alternative approaches for therapeutic modulation. Genome Med. (2016) 8:39. doi: 10.1186/s13073-01 6-0294-z

48. Tedijanto C, Olesen SW, Grad YH, Lipsitch M. Estimating the proportion of bystander selection for antibiotic resistance among potentially pathogenic bacterial flora. Proc Natl Acad Sci USA. (2018) 115:E11988-95. doi: $10.1073 /$ pnas. 1810840115

49. Kenyon CR, Delva W. It's the network, stupid: a population's sexual network connectivity determines its STI prevalence. F1000Research. (2018) 7:1880-7. doi: 10.12688/f1000research.17148.1

50. Reyniers T, Nöstlinger C, Laga M, De Baetselier I, Crucitti T, Wouters K, et al. Choosing between daily and event-driven pre-exposure prophylaxis: results of a Belgian PrEP demonstration project. J Acquir Immune Defic Syndr. (2018) 79:186-94. doi: 10.1097/QAI.0000000000001791

Conflict of Interest Statement: The author declares that the research was conducted in the absence of any commercial or financial relationships that could be construed as a potential conflict of interest.

Copyright (c) 2019 Kenyon. This is an open-access article distributed under the terms of the Creative Commons Attribution License (CC BY). The use, distribution or reproduction in other forums is permitted, provided the original author(s) and the copyright owner(s) are credited and that the original publication in this journal is cited, in accordance with accepted academic practice. No use, distribution or reproduction is permitted which does not comply with these terms. 